\title{
Trajectory of vitamin D status during pregnancy in relation to neonatal birth size and fetal survival: a prospective cohort study
}

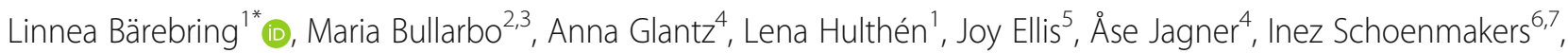
Anna Winkvist ${ }^{1}$ and Hanna Augustin ${ }^{1}$

\begin{abstract}
Background: We investigated the associations between vitamin D status in early and late pregnancy with neonatal small for gestational age (SGA), low birth weight (LBW) and preterm delivery. Furthermore, associations between vitamin D status and pregnancy loss were studied.

Methods: Serum 25-hydroxyvitamin D (25OHD) was sampled in gestational week $\leq 16$ (trimester 1 (T1), N=2046) and $>31$ (trimester $3(T 3), N=1816$ ) and analysed using liquid chromatography tandem mass spectrometry. Pregnant women were recruited at antenatal clinics in south-west Sweden at latitude $57-58^{\circ} \mathrm{N}$. Gestational and neonatal data were retrieved from medical records. Multiple gestations and terminated pregnancies were excluded from the analyses. SGA was defined as weight and/or length at birth $<2$ SD of the population mean and LBW as $<2500 \mathrm{~g}$. Preterm delivery was defined as delivery $<37+0$ gestational weeks and pregnancy loss as spontaneous abortion or intrauterine fetal death. Associations between neonatal outcomes and 25OHD at T1, T3 and change in 25OHD (T3-T1) were studied using logistic regression.

Results: $\mathrm{T1} 25 \mathrm{OHD}$ was negatively associated with pregnancy loss and $1 \mathrm{nmol} / \mathrm{L}$ increase in 25OHD was associated with $1 \%$ lower odds of pregnancy loss (OR 0.99, $p=0.046$ ). $T 325 \mathrm{OHD} \geq 100 \mathrm{nmol} / \mathrm{L}$ (equal to $40 \mathrm{ng} / \mathrm{ml}$ ) was associated with lower odds of SGA (OR 0.3, $p=0.031)$ and LBW (OR 0.2, $p=0.046)$, compared to vitamin D deficiency $(25 \mathrm{OHD}<30 \mathrm{nmol} / \mathrm{L}$, or $12 \mathrm{ng} / \mathrm{ml})$. Women with a $\geq 30 \mathrm{nmol} / \mathrm{L}$ increment in $25 \mathrm{OHD}$ from $\mathrm{T} 1$ to $\mathrm{T} 3$ had the lowest odds of SGA, LBW and preterm delivery.

Conclusions: Vitamin D deficiency in late pregnancy was associated with higher odds of SGA and LBW. Lower 25OHD in early pregnancy was only associated with pregnancy loss. Vitamin D status trajectory from early to late pregnancy was inversely associated with SGA, LBW and preterm delivery with the lowest odds among women with the highest increment in 25OHD. Thus, both higher vitamin D status in late pregnancy and gestational vitamin D status trajectory can be suspected to play a role in healthy pregnancy.
\end{abstract}

Keywords: Vitamin D, 25-hydroxyvitamin D, Small for gestational age, Low birth weight, Preterm delivery, Miscarriage, Intrauterine fetal death

\footnotetext{
* Correspondence: linnea.barebring@gu.se

${ }^{1}$ The Department of Internal Medicine and Clinical Nutrition, Sahlgrenska

Academy, University of Gothenburg, Box 459, 40530 Gothenburg, Sweden

Full list of author information is available at the end of the article
} 


\section{Background}

Placental pathology is often found in pregnancies complicated by intrauterine growth restriction, preeclampsia or intrauterine fetal death. It is associated with inadequate invasion of extravillous trophoblasts and inadequate angiogenesis, with insufficient conversion of arterial spiral arteries in the decidua [1]. These processes are complex and normal development is dependent on several factors. Vitamin D status (measured as 25-hydroxyvitamin D (25OHD)) of pregnant women has been inversely associated with adverse gestational outcomes and associated with uteroplacental dysfunction [2].

We have previously shown that higher vitamin D status in late pregnancy and larger increase in vitamin D status during pregnancy is associated with lower risk of preeclampsia [3]. Associations have also been shown between vitamin $\mathrm{D}$ insufficiency $(<50 \mathrm{nmol} / \mathrm{L})$ and increased risk of infant small for gestational age (SGA) and low birth weight (LBW) [4]. Circulating concentrations of $25 \mathrm{OHD}$ below 25 or $30 \mathrm{nmol} / \mathrm{L}$ have been associated with $50-300 \%$ increased odds of SGA, compared to higher concentrations [5-7]. Also, maternal 25OHD $<28 \mathrm{nmol} / \mathrm{L}$ in late, but not early, pregnancy have been associated with lower infant birth size and shorter gestational length in a smaller longitudinal study [8]. However, there is also limited evidence for a U-shaped association where high $25 \mathrm{OHD}$ concentrations $(>80 \mathrm{nmol} /$ L) may be related to higher risk of SGA [9]. Thus, the association between vitamin D status and neonatal birth size is not clear and warrants further investigation. A recent meta-analysis of 10 studies concluded that 25OHD concentrations $<50 \mathrm{nmol} / \mathrm{L}$ were associated with an approximately $30 \%$ increased risk of preterm delivery [10]. Bodnar et al. found that early pregnancy $25 \mathrm{OHD}$ concentrations $<75 \mathrm{nmol} / \mathrm{L}$ were associated with higher risk of both medically indicated and spontaneous preterm delivery [11]. Lower vitamin D status has also been associated with a medical history of recurrent miscarriage [12] but only two prospective studies have investigated this, without finding that lower 25OHD concentrations increases the risk of pregnancy loss $[5,13]$. Both these prospective studies had relatively few cases of pregnancy loss and may therefore have been insufficiently powered to study this association. Thus, the association between vitamin D status and risk of miscarriage needs further investigation.

To our knowledge, no previous study has related 25OHD concentration in both early and late pregnancy to neonatal outcomes related to placental dysfunction. Therefore, it has not been possible to ascertain whether the associations at implantation in early pregnancy differ from those during the fetal growth spurt in late pregnancy.

Our objectives were to study the associations between vitamin D status in both early and late pregnancy, as well as change in vitamin $\mathrm{D}$ status during pregnancy with neonatal SGA, LBW and preterm delivery. Associations between vitamin $\mathrm{D}$ status in early pregnancy and pregnancy loss were also studied.

\section{Methods}

The GraviD study is a prospective cohort study, conducted in parts of the Västra Götaland region in the southwest of Sweden, at latitude $57-58^{\circ} \mathrm{N}$ [3]. Pregnant women were recruited from gestational week 4 during fall 2013 and spring 2014, when registering at one of the participating antenatal care units. The only exclusion criterion was pregnancy exceeding 16 gestational weeks at inclusion. Gestational age at delivery and data collection was determined by routine ultrasound in the second trimester, but gestational age at inclusion was based on the date of the last menstrual period. Women who terminated the pregnancy $(N=31)$, were lost to follow-up $(N=13)$ or carried more than one foetus $(N=26)$ were excluded from the present analyses. Pregnancy termination was mostly due to fetal malformations. Women who were lost to follow-up had moved and their medical records could not be retrieved. This study was conducted according to the Declaration of Helsinki and all procedures were approved by the Regional Ethics Committee in Gothenburg, Sweden. Written informed consent was obtained from all participants.

\section{Outcomes}

SGA was defined as either weight or length at birth below 2 SD of the gender-specific population mean [14]. LBW was defined as weight at birth $<2500 \mathrm{~g}$. Preterm delivery was defined as delivery before gestational week +days $37+0$, including both induced and spontaneous preterm delivery. Pregnancy loss was defined as both spontaneous abortions from gestational week 4 and intrauterine fetal death (IUFD). Late pregnancy loss was defined as spontaneous abortion at gestational week $\geq 14$ +0 , including IUFD. IUFD was defined as pregnancy loss at gestational week $\geq 22+0$. Pregnancy loss before gestational week 22 was based on self-report data and medical records to verify miscarriage were not available. Information on IUFD was collected from medical records from the obstetrics care.

\section{Exposure}

Maternal blood samples were collected from each participant at two time points; before gestational week 16 (predominantly week 8-12, first trimester, T1) and after gestational week 31 (predominantly week $32-35$, third trimester, T3). Non-fasting venous blood samples were drawn in gel serum separating tubes and centrifuged for $10 \mathrm{~min}$ within $2 \mathrm{~h}$ of sampling. Serum was stored at $-70{ }^{\circ} \mathrm{C}$ until analysis of $25 \mathrm{OHD}$. Analyses were performed by liquid chromatography tandem mass spectrometry (LC-MS/MS, 
Mass spectrometer API 4000) by the clinical chemistry laboratory in Region Skåne, Sweden, certified by the Vitamin D External Quality Assessment Scheme [15]. The LC-MS/ MS method has a measuring range of $6-450 \mathrm{nmol} / \mathrm{L}$ for $25 \mathrm{OHD}_{3}$ and of $6-225 \mathrm{nmol} / \mathrm{L}$ for $25 \mathrm{OHD}_{2}$. The interassay coefficient of variation is $6 \%$ at $40 \mathrm{nmol} / \mathrm{L}$ for both $25 \mathrm{OHD}_{3}$ and $25 \mathrm{OHD}_{2}$. Sampling and laboratory analyses have been described previously [3]. Maternal serum T1 and T3 samples were analysed in sequence. At T1 and T3, participants answered questionnaires regarding lifestyle factors and background data. Neonatal and gestational data were obtained from antenatal and obstetrics medical records. Information on BMI at T1 and season of conception (December-May or June-November) was obtained from the medical records. Season was coded as a binary variable, since this explained $30 \%$ of vitamin D status in a previous study in pregnant women at the same latitude [16]. Data on education level ( $\leq$ primary level, secondary level or university level) and origin (continent of birth: Northern Europe, Continental Europe, America, Asia, Africa) were collected from study questionnaires.

\section{Statistical analysis}

Concentrations of 25OHD at T1 and T3 were used as categorical variables, grouped into $25 \mathrm{OHD}<30$ (used as reference category), 30-49.9, 50-74.9, 75-99.9 and $\geq$ $100 \mathrm{nmol} / \mathrm{L}$. These groups were chosen to study whether vitamin D insufficiency $(30-50 \mathrm{nmol} / \mathrm{L})$, sufficiency $(\geq$ $50 \mathrm{nmol} / \mathrm{L}$ ) or high status ( $\geq 75$ or $\geq 100 \mathrm{nmol} / \mathrm{L}$ ) was associated with the outcomes studied, compared to vitamin D deficiency $(<30 \mathrm{nmol} / \mathrm{L})$. Quartiles were not used because the distribution of $25 \mathrm{OHD}$ was different at $\mathrm{T} 1$ and $\mathrm{T} 3 \mathrm{~T} 1$ $25 \mathrm{OHD}$ was also investigated as a continuous variable as there were few cases in some categories. Change in 25OHD was calculated as the difference between T3 and T1 (T3-T1) and was coded into 3 groups: decrease in $25 \mathrm{OHD}(\leq 0 \mathrm{nmol} / \mathrm{L})$, small increase $(0.1-29.99 \mathrm{nmol} / \mathrm{L})$ or large increase $(\geq 30 \mathrm{nmol} / \mathrm{L})$.

Multivariable logistic regression analyses of the outcome variables SGA, LBW and preterm delivery were performed with $25 \mathrm{OHD}$ at $\mathrm{T} 1, \mathrm{~T} 3$ and change in $25 \mathrm{OHD}$ during pregnancy as the independent variables. For SGA, appropriate for gestational age and large for gestational age were combined as reference. Potential confounders for the associations studied were identified using directed acyclic graphs (www.dagitty.net) [17]. Variables BMI, season of conception, education level and origin were identified and included in the final models. Tobacco use and vitamin D supplement use were also investigated as potential confounders but did not show any confounding effect and were thus not included in the final models. In the multivariable analysis with change in $25 \mathrm{OHD}$ as the independent variable, T1 25OHD was also included as a confounder. Correlation between continuous T1 25OHD and continuous change in 25OHD was low $(r=-0.22, p$ $<0.001)$ and it was therefore considered acceptable to include both variables in the same model. Multivariable logistic regression analysis of the outcome pregnancy loss was also performed with 25OHD at T1 (continuous and categorical) as independent variable. These models were adjusted for BMI, season of conception, education level, origin and gestational age at registration to antenatal care (based on last menstrual period). Unadjusted logistic regression analysis was used to assess the association between T1 25OHD (<30 nmol/L; no/yes) and IUFD. Here, no confounders were included due to the small number of cases $(N=9)$. Significance was accepted at $p<0.05$. Computer software IBM SPSS Statistics version 22.0 was used for all statistical analyses.

\section{Results}

In total, 2052 women were included in this study, 2046 with a blood sample at T1 and 1816 at T3 (Fig. 1). In total, 1810 women had samples collected both in T1 and T3. Characteristics of the women and the live born infants are shown in Table 1. Mean infant birth weight was 3542 (538) grams and mean gestational age at delivery was 280 (12.4) days. Mean (SD) maternal 25OHD at $\mathrm{T} 1$ was $64(24.4) \mathrm{nmol} / \mathrm{L}$ and $75(34.4) \mathrm{nmol} / \mathrm{L}$ at T3. At T1, 10\% had 25OHD concentrations $<30 \mathrm{nmol} / \mathrm{L}$ and $9 \%$ at T3 (Table 2).

\section{Birth size and preterm delivery}

In total, 93 (4.5\%) infants were born SGA. Of the SGA deliveries, 37 were SGA by weight and 56 by length. Also, 58 (2.8\%) infants had LBW, while 78 (3.8\%) infants were delivered preterm (Table 1). Of those who delivered preterm, 10 delivered before gestational week 31 and thus before the T3 blood sample could be drawn.

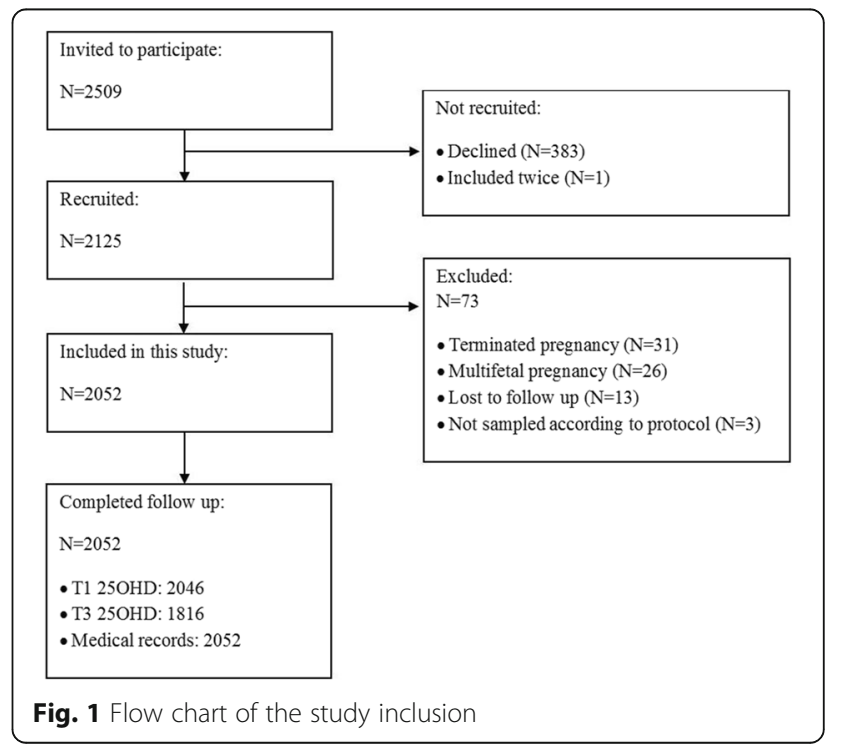


Table 1 Characteristics of the pregnant women and their infants at birth

\begin{tabular}{llr}
\hline & Mean & SD \\
\hline Birth weight (grams) $^{\text {a }}$ & 3542 & 537.7 \\
Birth length (cm) $^{\text {a }}$ & 50 & 2.3 \\
Gestational age at delivery (days) & 280 & 12.4 \\
Gestational age T1 (days) & 76 & 13.8 \\
Gestational age T3 (days) & 234 & 12.9 \\
Maternal s-25OHD T1 (nmol/L) & 64 & 24.4 \\
Maternal s-25OHD T3 (nmol/L) & 75 & 34.4 \\
& $\mathrm{~N}(\%)$ & \\
Male gender of infant ${ }^{\mathrm{a}}$ & $976(49.8)$ & \\
Small for gestational age (weight or length) & $93(4.5)$ & \\
Low birth weight $(<2500 \mathrm{~g})$ & $58(2.8)$ & \\
Preterm delivery (<37 weeks) & $78(3.8)$ & \\
Spontaneous preterm delivery (<37 weeks) & $55(2.7)$ & \\
Pregnancy loss & $97(4.7)$ & \\
Intrauterine fetal death ( $\geq 22$ weeks) & $9(0.5)$ & \\
\hline
\end{tabular}

$T 1$ first trimester, $T 3$ third trimester, $250 \mathrm{HD} 25$-hydroxyvitamin D

aLive born infants only
Among these women, one (10\%) had 25OHD concentration $<30 \mathrm{nmol} / \mathrm{L}$ in $\mathrm{T} 1$.

There were no associations between the predictor $\mathrm{T} 1$ 25OHD and the outcomes SGA, LBW and preterm delivery (Table 3). Women with T3 $25 \mathrm{OHD} \geq 100 \mathrm{nmol} / \mathrm{L}$ had a lower OR for SGA (OR $=0.32, p=0.031)$ compared to those with vitamin $\mathrm{D}$ deficiency $(<30 \mathrm{nmol} / \mathrm{L})$. Women with $\mathrm{T} 3$ $25 \mathrm{OHD}$ concentrations $\geq 100 \mathrm{nmol} / \mathrm{L}$ and $30-50 \mathrm{nmol} / \mathrm{L}$ had lower OR for LBW $(\mathrm{OR}=0.22, p=0.046$ and $\mathrm{OR}=$ $0.07, p=0.017$, respectively), compared to vitamin $\mathrm{D}$ deficiency. However, T3 25OHD was not significantly associated with preterm delivery. The results were not meaningfully affected by additional adjustment for tobacco use or vitamin D supplementation or by excluding cases of preeclampsia (data not shown).

Vitamin D status trajectory between T1 and T3 was inversely related to SGA, preterm and LBW (Table 3). Compared to women with a large increase in 25OHD $(\geq 30 \mathrm{nmol} / \mathrm{L})$, women with a decrease in $25 \mathrm{OHD}$ had significantly higher OR of having a child born SGA (OR $=3.7, p=0.002)$, with LBW (OR $=4.7, p=0.014)$ as well as a trend toward higher odds of preterm delivery $(\mathrm{OR}=$ $2.9, p=0.061)$. Women with a small increase in 25OHD $(0-30 \mathrm{nmol} / \mathrm{L})$ had significantly higher OR of SGA (OR $=2.6, p=0.019)$ and preterm delivery $(\mathrm{OR}=2.9, p=$ 0.047 ) as well as a trend toward significance for LBW

Table 2 The pregnant women's vitamin D status in the first (T1) and third (T3) trimester of pregnancy, and their characteristics (mean or percent) grouped by $25 \mathrm{OHD}$ concentration in (T1)

\begin{tabular}{|c|c|c|c|c|c|}
\hline T1 25OHD (nmol/L) & $<30$ & $30-49.9$ & $50-74.9$ & $75-99.9$ & $\geq 100$ \\
\hline $\mathrm{N}$ & 198 & 291 & 788 & 565 & 125 \\
\hline$\%$ & 10.1 & 14.8 & 40.1 & 28.7 & 6.4 \\
\hline BMI T1 $\left(\mathrm{kg} / \mathrm{m}^{2}\right)$ & 25.1 & 24.9 & 24.4 & 23.9 & 24.4 \\
\hline Age T1 (years) & 29.4 & 30.9 & 31.6 & 32.0 & 32.4 \\
\hline Born in Sweden (\%) & 14.1 & 54.6 & 83.3 & 87.8 & 93.5 \\
\hline Tobacco use T1 (\%) & 6.8 & 4.6 & 4.0 & 4.6 & 1.6 \\
\hline Nulliparous T1 (\%) & 34.8 & 42.0 & 42.6 & 43.1 & 42.7 \\
\hline Vitamin D supplement use T1 (\%) & 10.6 & 28.2 & 45.2 & 56.6 & 62.9 \\
\hline University level education (\%) & 32.3 & 54.9 & 61.3 & 67.3 & 66.1 \\
\hline Small for gestational age (\%) & 5.7 & 4.2 & 5.3 & 4.1 & 4.0 \\
\hline Preterm delivery (\%) & 4.1 & 5.2 & 3.8 & 3.9 & 1.6 \\
\hline Low birth weight (\%) & 3.0 & 4.5 & 3.1 & 2.1 & 1.6 \\
\hline Pregnancy loss (\%) & 6.3 & 5.6 & 4.5 & 5.1 & 0 \\
\hline T3 25OHD (nmol/L) & $<30$ & $30-49.9$ & $50-74.9$ & $75-99.9$ & $\geq 100$ \\
\hline $\mathrm{N}$ & 163 & 330 & 473 & 409 & 443 \\
\hline$\%$ & 9.0 & 18.2 & 26.0 & 22.5 & 24.4 \\
\hline Small for gestational age (\%) & 7.5 & 5.5 & 4.7 & 4.6 & 2.3 \\
\hline Preterm delivery (\%) & 4.3 & 1.2 & 3.6 & 2.2 & 1.6 \\
\hline Low birth weight (\%) & 4.3 & 0.3 & 2.7 & 1.2 & 1.4 \\
\hline
\end{tabular}

$T 1$ first trimester, $T 3$ third trimester, $25 \mathrm{OHD} 25$-hydroxyvitamin D 
Table 3 Association between vitamin D status in pregnancy with birth size and pregnancy loss (adjusted logistic regression analysis)

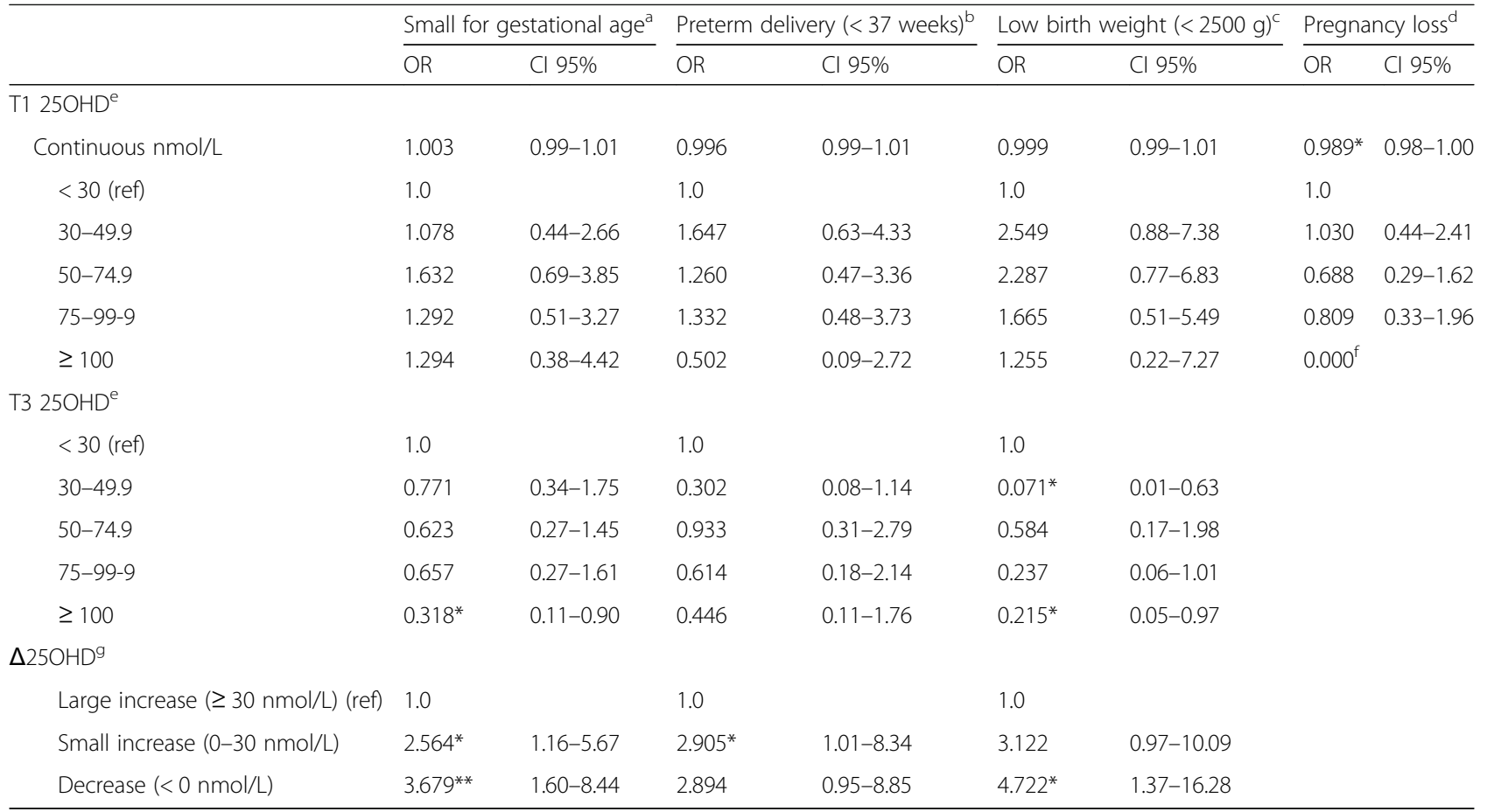

T1 first trimester, $T 3$ third trimester, 25OHD 25-hydroxyvitamin D

${ }^{*} p<0.05{ }^{* *} p<0.01$

a Models include 92 (T1) and 80 (T3 and $\triangle 250 H D$ ) cases of small for gestational age

${ }^{\mathrm{b}}$ Models include 77 (T1) and 43 (T3 and $\triangle 25 \mathrm{OHD}$ ) cases of preterm delivery

${ }^{c}$ Models include 57 (T1) and 31 (T3 and $\triangle 25 \mathrm{OHD}$ ) cases of low birth weight

${ }^{\mathrm{d}}$ Models include 96 cases of pregnancy loss

eAdjusted for education, origin, season of conception and BMI at T1. Pregnancy loss models are also adjusted for gestational age at registration for antenatal care f No case of pregnancy loss in category

${ }^{\mathrm{g}}$ Adjusted for education, origin, season of conception, BMI at T1 and 25OHD at T1

$(\mathrm{OR}=3.1, p=0.056)$, compared to women with a large increase. Neither of these results changed after adjustment for tobacco use and vitamin D supplementation at T3 or after excluding cases of preeclampsia.

\section{Pregnancy loss}

The total rate of pregnancy loss was $4.7 \%$ and the rate of late pregnancy loss (gestational week $\geq 14+0$ ) was $1.5 \%$ (Table 1). There were nine cases of IUFD at gestational week $\geq 22+0$. Overall, lower T1 25OHD (as a continuous but not categorical variable) was associated with pregnancy loss (Table 3). In unadjusted analysis, T1 25OHD was associated with IUFD and women with $25 \mathrm{OHD}<30 \mathrm{nmol} / \mathrm{L}$ at $\mathrm{T} 1$ had more than fourfold higher odds of IUFD (OR $=4.52, p=0.034)$.

\section{Discussion}

We found that higher vitamin D status among women in late, but not early, pregnancy was associated with lower probability of SGA and LBW. Vitamin D status trajectory during pregnancy was inversely associated with SGA, LBW and preterm delivery. Women with an increase in $25 \mathrm{OHD} \geq 30 \mathrm{nmol} / \mathrm{L}$ from $\mathrm{T} 1$ to $\mathrm{T} 3 \mathrm{had}$ the lowest odds of SGA, LBW and preterm delivery. We also found that lower vitamin D status in early pregnancy was related to pregnancy loss.

Previous findings from the GraviD study show lower odds of preeclampsia among women with a large increase $(\geq 30 \mathrm{nmol} / \mathrm{L})$ in $25 \mathrm{OHD}$ [3]. As SGA, LBW and preterm delivery are related to preeclampsia; these results are consistent with the current findings. However, excluding preeclampsia cases from the analysis did not change the results. Thus, preeclampsia does not seem to mediate the associations between vitamin D status trajectory and neonatal birth size or preterm birth. We have previously shown that the determinants of season-corrected change in 25OHD during pregnancy include origin, sun-exposure and dietary as well as supplementary vitamin D intake [18]. Supplements containing vitamin D were used by $43 \%$ in $\mathrm{T} 1$ and 39\% in T3- mainly multivitamins [19]. As the results between change in 25OHD and neonatal birth size and preterm delivery remained after adjustment for season of conception, origin and vitamin D supplementation, vitamin D status trajectory can be suspected to play a role in healthy pregnancy. Vitamin D has been shown to facilitate the transport of nutrients across the placenta [20, 21], 
which could contribute to fetal growth [22]. Vitamin D status could also facilitate fetal development by regulating placental inflammation [23]. It is also possible that the associations between fetal growth and maternal vitamin D status are due to residual confounding or reverse causation. Whether vitamin D metabolism is altered in placental dysfunction in unclear and warrants further investigation.

Our results indicates that $25 \mathrm{OHD}$ at T3 but not at T1 is associated with SGA and LBW, despite better statistical power at $\mathrm{T} 1$ due to more $25 \mathrm{OHD}$ samples and subsequently more cases of SGA, LBW and preterm birth. This finding is partly consistent with previous studies where $25 \mathrm{OHD}$ concentrations of $25-30 \mathrm{nmol} / \mathrm{L}$ were associated with higher probability of SGA [5-7]. The study by Burris et al. [6] sampled women in gestational week 26-28, and found a higher OR for SGA than the two studies that sampled women in T1. This could be interpreted as support of our finding that late rather than early pregnancy vitamin D status is the stronger predictor of fetal growth restriction. Our results also concur with findings by Morley et al. that late but not early pregnancy 25OHD was related to gestational length and neonatal birth size [8]. Our results could also indicate that it takes time for changes in vitamin $\mathrm{D}$ metabolism to manifest as changes in circulating 25OHD. We did not see a U-shaped association between early pregnancy vitamin D status and SGA, as previously indicated [9]. We found the lowest odds of SGA among women with the highest T3 25OHD concentration, $\geq 100 \mathrm{nmol} / \mathrm{L}$. A total of $25 \%$ of the women had $25 \mathrm{OHD}$ concentrations $\geq 100 \mathrm{nmol} / \mathrm{L}$ at T3. Sampling was evenly distributed across the seasons and time of year cannot explain the high proportion with high 25OHD concentrations. Also, vitamin D supplements were used by almost half of the women but most (88\%) used multivitamins containing 5-10 $\mu$ g of vitamin D3. Supplementation can therefore only partly explain the large proportion with high vitamin D status, and other likely contributors are pregnancy associated endocrine changes and possibly lifestyle factors.

To our knowledge, ours is one of the first studies to find associations between vitamin D status and pregnancy loss. The 25OHD concentration has been shown to have immunological effects in women with a history of recurrent pregnancy loss [12]. Two previous studies have investigated but not found any association between $25 \mathrm{OHD}$ and miscarriage $[5,13]$. In those studies, 25OHD was used as a categorical variable. One earlier study, from Denmark, found that lower vitamin D status in early pregnancy was associated with pregnancy loss in the first trimester [24]. We found that pregnancy loss was associated with lower 25OHD when expressed on a continuous but not categorical scale. As most women with high vitamin D status were born in Sweden and thus more likely to be familiar with the Swedish health- care system, it is possible that they registered earlier for antenatal care and were more likely to report pregnancy loss. However, the models were adjusted for gestational age at registration for antenatal care. Despite few cases of IUFD in the GraviD cohort, our results suggest that vitamin D deficiency in early pregnancy may be linked to IUFD. These results need confirmation, preferably by adjusted statistical analysis as confounding can be expected, which was not possible in our present study due to few cases.

Strengths of this study are that the GraviD cohort is representative of the general pregnant population in terms of origin, education, parity, BMI and tobacco use [25], which increases the external validity of the findings. Also, gestational age at delivery was estimated by routine ultrasound, which is considered more accurate than dating by last menstrual period [26]. A limitation of this study is that information on pregnancy loss, except IUFD, was self-reported. The information on pregnancy loss is likely correct, albeit without conclusive information on the time of fetal demise. Survival analysis could therefore not be performed, as time to event data were missing. In addition, vitamin D status among women who terminated the pregnancy was not assessed. Data on SGA, LBW and preterm delivery were collected from obstetrics charts based on standardized measures. Since there were few cases of SGA based on birth weight alone, the definition of SGA used in this study was based on birth weight and/or length. Among the SGA cases defined by length only, most were close to also meeting the SGA definition for birth weight. Therefore, the definition used in this study is likely to be relevant. Another limitation is that data on physical activity during pregnancy was only available for a subset of the women and was therefore not used.

While the GraviD study indicates an association between T3 25OHD and consequences of placental insufficiency, change in 25OHD during pregnancy might be a stronger predictor of placental dysfunction, as it is associated with SGA, LBW and preterm delivery. The lowest odds of SGA and LBW are found among women with the largest increments in $25 \mathrm{OHD}(\geq 30 \mathrm{nmol} / \mathrm{L})$. Interestingly, vitamin D status at T1- around the time the maternal blood flow of the placenta is fully developed- is only related to pregnancy loss.

\section{Conclusion}

In conclusion, lower early pregnancy $25 \mathrm{OHD}$ was associated with pregnancy loss. High vitamin D status in late, but not early, pregnancy was associated with lower odds of SGA and LBW. Change in 25OHD during pregnancy was associated with SGA, LBW and preterm delivery, with the lowest odds for women with an increment in 25OHD $\geq$ $30 \mathrm{nmol} / \mathrm{L}$. Both higher late pregnancy vitamin D status and gestational vitamin $\mathrm{D}$ status trajectory can be suspected to play a role in healthy pregnancy. 


\section{Abbreviations}

25OHD: 25-hydroxyvitamin D; IUFD: Intrauterine fetal death; LBW: Low birth weight; SGA: Small for gestational age; T1: First trimester of pregnancy; T3: Third trimester of pregnancy

\section{Acknowledgements}

The authors would like to thank the women who participated in the study, as well as the midwives and nurses whose contributions were pivotal in the realization of the study.

\section{Funding}

This research was funded by the Swedish Research Council for Health, Working Life and Welfare (HA, Forte, Dnr 2012-0793) and Regional Research and Development grants (MB, FoU, Dnr VGFOUREG-388201 and VGFOUREG-229331). Inez Schoenmakers was funded by the Medical Research Council, programme U105960371. The funders had no role in study design, data collection and analysis, decision to publish, or preparation of the manuscript.

\section{Availability of data and materials}

Data cannot be made freely available as they are subject to secrecy in accordance with the Swedish Public Access to Information and Secrecy Act (Offentlighets- och sekretesslagen, OSL, 2009:400), but can be made available to researchers upon reasonable request, subject to a review of secrecy. Requests for data should be made to corresponding author.

\section{Authors' contributions}

HA MB AG JE LH conceived and designed the study. LB AJ HA collected the data. LB analyzed the data. LB MB AG AW AJ JE LH IS HA wrote the paper and all authors have contributed substantially in drafting and revising the manuscript. All authors read and approved the final manuscript.

\section{Ethics approval and consent to participate}

All participants provided written informed consent and all procedures were approved by the Regional Ethics Committee in Gothenburg (Dnr 897-11 and T439-13).

\section{Consent for publication}

Not applicable.

\section{Competing interests}

The authors declare that they have no competing interests.

\section{Publisher's Note}

Springer Nature remains neutral with regard to jurisdictional claims in published maps and institutional affiliations.

\section{Author details}

${ }^{1} T$ The Department of Internal Medicine and Clinical Nutrition, Sahlgrenska Academy, University of Gothenburg, Box 459, 40530 Gothenburg, Sweden. ${ }^{2}$ Södra Älvsborg Hospital, Borås, Sweden. ${ }^{3}$ The Department of Obstetrics and Gynecology, Sahlgrenska Academy, University of Gothenburg, Gothenburg, Sweden. ${ }^{4}$ Department of Antenatal Care, Närhälsan, Primary Care, Gothenburg, Sweden. ${ }^{5}$ Department of Antenatal Care, Närhälsan, Primary Care, Södra, Bohuslän, Sweden. ${ }^{6}$ MRC Human Nutrition Research, Nutrition and Bone Health Group, Cambridge, UK. ${ }^{7}$ The Department of Medicine, Faculty of Medicine and Health Sciences, University of East Anglia, Norwich, UK.

Received: 18 October 2016 Accepted: 1 February 2018 Published online: 13 February 2018

\section{References}

1. Hacker NF, Gambone JC, Hobel CJ. Hacker and Moore's essentials of obstetrics and gynecology. 5th ed. Philadelphia: Saunders Elsevier; 2010.

2. Kiely ME, Zhang JY, Kinsella M, Khashan AS, Kenny LC. Vitamin D status is associated with uteroplacental dysfunction indicated by pre-eclampsia and small-for-gestational-age birth in a large prospective pregnancy cohort in Ireland with low vitamin D status. Am J Clin Nutr. 2016:104(2):354-61.

3. Bärebring L, Bullarbo M, Glantz A, Leu Agelii M, Jagner A, Ellis J, et al. Preeclampsia and blood pressure trajectory during pregnancy in relation to vitamin D status. PLoS One. 2016;11(3):e0152198.
4. Chen YH, Fu L, Hao JH, Yu Z, Zhu P, Wang H, et al. Maternal vitamin D deficiency during pregnancy elevates the risks of small for gestational age and low birth weight infants in Chinese population. J Clin Endocrinol Metab. 2015:100(5):1912-9.

5. Schneuer FJ, Roberts CL, Guilbert C, Simpson JM, Algert CS, Khambalia AZ, et al. Effects of maternal serum 25-hydroxyvitamin D concentrations in the first trimester on subsequent pregnancy outcomes in an Australian population1-3. Am J Clin Nutr. 2014;99(2):287-95.

6. Burris HH, Rifas-Shiman SL, Camargo CA, Litonjua AA, Huh SY, Rich-Edwards JW, et al. Plasma 25-hydroxyvitamin D during pregnancy and small-for-gestational age in black and white infants. Ann Epidemiol. 2012;22(8):581-6.

7. Leffelaar ER, Vrijkotte TGM, Van Eijsden M. Maternal early pregnancy vitamin D status in relation to fetal and neonatal growth: results of the multi-ethnic Amsterdam born children and their development cohort. Br J Nutr. 2010;104(1):108-17.

8. Morley R, Carlin JB, Pasco JA, Wark JD. Maternal 25-hydroxyvitamin D and parathyroid hormone concentrations and offspring birth size. J Clin Endocrinol Metab. 2006;91(3):906-12.

9. Bodnar LM, Catov JM, Zmuda JM, Cooper ME, Parrott MS, Roberts JM, et al. Maternal serum 25-hydroxyvitamin D concentrations are associated with smallfor-gestational age births in white women. J Nutr. 2010;140(5):999-1006.

10. Qin LL, Lu FG, Yang SH, Xu HL, Luo BA. Does maternal vitamin D deficiency increase the risk of preterm birth: a meta-analysis of observational studies. Nutrients. 2016:8:5

11. Bodnar LM, Platt RW, Simhan HN. Early-pregnancy vitamin D deficiency and risk of preterm birth subtypes. Obstet Gynecol. 2015;125(2):439-47.

12. Ota K, Dambaeva S, Han AR, Beaman K, Gilman-Sachs A, Kwak-Kim J. Vitamin D deficiency may be a risk factor for recurrent pregnancy losses by increasing cellular immunity and autoimmunity. Hum Reprod. 2014;29(2):208-19.

13. Zhou J, Su L, Liu M, Liu Y, Cao X, Wang Z, et al. Associations between 25hydroxyvitamin $D$ levels and pregnancy outcomes: a prospective observational study in southern China. Eur J Clin Nutr. 2014;68(8):925-30.

14. Clayton PE, Cianfarani S, Czernichow P, Johannsson G, Rapaport R, Rogol AD. Consensus statement: management of the child born small for gestational age through to adulthood: a consensus statement of the international societies of pediatric endocrinology and the growth hormone research society. J Clin Endocrinol Metab. 2007;92(3):804-10.

15. Metodbeskrivning S-25-OH Vitamin D3, S-25-OH Vitamin D2, Malmö [Internet]. 2014 [cited 2015-08-19]. Available from: http://analysportalenlabmedicin.skane.se/pics/Labmedicin/Verksamhetsomr\%E5den/ Klinisk\%20kemi/Analyser/Skane/S-25-OH\%20Vitamin\%20D3,\%20S-25OH\%20Vitamin\%20D2.pdf.

16. Brembeck $\mathrm{P}$, Winkvist $\mathrm{A}$, Olausson $\mathrm{H}$. Determinants of vitamin D status in pregnant fair-skinned women in Sweden. Br J Nutr. 2013;110(5):856-64.

17. Textor J, Hardt J, Knuppel S. DAGitty: a graphical tool for analyzing causal diagrams. Epidemiology. 2011;22(5):745

18. Bärebring L, Schoenmakers I, Glantz A, Hulthén L, Jagner $\AA$, Ellis J, et al. Vitamin D status during pregnancy in a multi-ethnic populationrepresentative Swedish cohort. Nutrients. 2016 22:8(10):655.

19. Bärebring L, Mullally D, Glantz A, Elllis J, Hulthen L, Jagner A, et al. Sociodemographic factors associated with dietary supplement use in early pregnancy in a Swedish cohort. Br J Nutr 2017:1-6.

20. Chen YY, Powell TL, Jansson T. 1,25-Dihydroxy vitamin D3 stimulates system a amino acid transport in primary human trophoblast cells. Mol Cell Endocrinol. 2017:442:90-7

21. Cleal JK, Day PE, Simner CL, Barton SJ, Mahon PA, Inskip HM, et al. Placental amino acid transport may be regulated by maternal vitamin $D$ and vitamin D-binding protein: results from the Southampton Women's survey. $\mathrm{Br}$ J Nutr. 2015;113(12):1903-10

22. Cetin I. Placental transport of amino acids in normal and growth-restricted pregnancies. Eur J Obstet Gynecol Reprod Biol. 2003;110(Suppl 1):S50-4.

23. Liu NO, Kaplan AT, Lagishetty V, Ouyang YB, Ouyang Y, Simmons CF, et al. Vitamin D and the regulation of placental inflammation. J Immunol. 2011;186(10):5968-74.

24. Andersen LB, Jorgensen JS, Jensen TK, Dalgard C, Barington T, Nielsen J, et al. Vitamin $D$ insufficiency is associated with increased risk of first-trimester miscarriage in the Odense child cohort. Am J Clin Nutr. 2015;102(3):633-8.

25. OFFICIAL STATISTICS OF SWEDEN. Pregnancies, deliveries and newborn infants the Swedish medical birth register 1973-2014; 2015. p. 1400-3511.

26. Dietz PM, England LJ, Callaghan WM, Pearl M, Wier ML, Kharrazi MA Comparison of LMP-based and ultrasound-based estimates of gestational age using linked California livebirth and prenatal screening records. Paediatr Perinat Epidemiol. 2007;21(Suppl 2):62-71. 